\title{
Antiretroviral resistance mutations in human immunodeficiency virus type 1 infected patients enrolled in genotype testing at the Central Public Health Laboratory, São Paulo, Brazil: preliminary results
}

\author{
Rosangela Rodrigues $/^{+}$, Carla Maria Pasquareli Vazquez*, Jeova Keny Colares**, \\ Renata Marconi Custodio, Francisco Bonásser Filho*, Lenice do Rosário Souza***, \\ Maria Clara Gianna****, Cristiano Corrêa de Azevedo Marques, \\ Luís Fernando de Macedo Brígido*****
}

\begin{abstract}
Laboratório de Retrovírus, Instituto Adolfo Lutz, Av. Dr. Arnaldo 355, 01246-902 São Paulo, SP, Brasil *Instituto de Infectologia Emílio Ribas, São Paulo, SP, Brasil **Hospital das Clínicas, Universidade de Ribeirão Preto, USP, Ribeirão Preto, SP, Brasil ***Faculdade de Medicina, Unesp, Botucatu, SP, Brasil ****Centro de Referência e Treinamento em DST/AIDS, São Paulo, SP, Brasil *****Programa Nacional de DST/AIDS, Ministério da Saúde, Brasília, DF, Brasil
\end{abstract}

Antiretroviral resistance mutations (ARM) are one of the major obstacles for pharmacological human immunodeficiency virus (HIV) suppression. Plasma HIV-1 RNA from 306 patients on antiretroviral therapy with virological failure was analyzed, most of them (60\%) exposed to three or more regimens, and $28 \%$ of them have started therapy before 1997. The most common regimens in use at the time of genotype testing were AZT/3TC/nelfinavir, 3TC/D4T/ nelfinavir and AZT/3TC/efavirenz. The majority of ARM occurred at protease (PR) gene at residue L90 (41\%) and V82 (25\%); at reverse transcriptase (RT) gene, mutations at residue M184 (V/I) were observed in $64 \%$. One or more thymidine analogue mutations were detected in $73 \%$. The number of ARM at PR gene increased from a mean of four mutations per patient who showed virological failure at the first ARV regimens to six mutations per patient exposed to six or more regimens; similar trend in RT was also observed. No differences in ARM at principal codon to the three drug classes for HIV-1 clades B or F were observed, but some polymorphisms in secondary codons showed significant differences. Strategies to improve the cost effectiveness of drug therapy and to optimize the sequencing and the rescue therapy are the major health priorities.

Key words: human immunodeficiency virus - diversity - antiretroviral resistance - Brazil

Antiretroviral therapy (ARV) has changed the scenario of the acquired immunodeficiency syndrome (AIDS) epidemic in Brazil (Casseb et al. 1999, Marins et al. 2003). Virus resistance to medication (Shafer et al. 1994, Larder \& Kemp 1989, D’Aquila et al. 2002) has been a major challenge to the long term success of therapy. However, genotypic information may orient the rescue ARV therapy (Clevenbergh et al. 2000). In 1999, the Adolfo Lutz Institute (IAL), with the support from the São Paulo State STD/ AIDS Program, has initiated the monitoring of HIV resistance and, in 2000, the use of genotype testing with salvage therapy recommendation to support clinical decision in patients with advanced disease has been established in a limited scale. Since November 2001, the IAL integrated the National Genotyping Network, which was organized to provide genotype testing for patients with treatment failure to the first protease inhibitors regimens. The Laboratory has also provided this assay for cases from judicial mandate and continued to provide the tests for patients presenting more advanced disease, generally

+Corresponding author. E-mail: rohc@usp.br

Received 7 July 2004

Accepted 15 December 2004 indicated for testing according to the evaluation provided by State Genotype Committee.

In the present study we analyzed the cumulative information from these sources to assess a preliminary data on resistance to ARV therapy.

\section{MATERIALS AND METHODS}

In the study, we included sequence analyses performed in samples from 306 patients, who had signed the written informed consent, with available information on ARV regimens, referred to the Retrovirus Laboratory, during the period from December 2001 to May 2003. Consecutive blood samples from patients referred from the Collaborative Clinical Centers with clinical indication for genotype testing were received and processed. Research Ethical Committee of Adolfo Lutz Institute approved this study.

Plasma was separated by centrifugation (4000 RPM for $20 \mathrm{~min}$ ) and preserved at $-70^{\circ} \mathrm{C}$ until use. The Viroseq (ABI, US) genotyping kit was used and performed according to manufacture's instructions. Alternatively, plasma virions were concentrated by centrifugation $(22,000$ $\mathrm{x} g$ for $90 \mathrm{~min}$ ). Viral RNA was extracted from plasma using the Trizol LS Reagent (Gibco, US) according to the procedure described in manufacturer's instructions. Reverse transcription of plasma HIV RNA was performed with 200 U of SuperScript II enzyme, (Life Technologies, US), 300 
ng of random hexamer primers (Life Technologies), 0.5 $\mathrm{mM}$ of each deoxynucleoside triphosphate, $7.5 \mathrm{U}$ of RNAse inhibitor (Life Technologies), and $10 \mathrm{mM}$ of dithiothreitol in a final volume of $20 \mu \mathrm{l}$, at $42^{\circ} \mathrm{C}$ for $90 \mathrm{~min}$ in reaction buffer. During the first PCR, for the polymerase gene, the DNA was amplified with the following pair of primers (position relative to $\mathrm{HXB} 2$, Accession number K03455): 5'-CAGAGCCAACAGCCCCACCA-3' (forward; position 2147-2166) and 5'-TTCCCCACTAACTT CTGTATGTCATTGACA-3' (reverse; position 5745-5776). The protease and reverse transcriptase genes were amplified by a second PCR (nested) using following primer pairs, respectively: 5'-TAACTCCCTCTCAGAA GCAGGAGCCG-3' (forward; position 2201-2226), 5'CCATTCCTGGCTTTAATTTTACTGGTA-3' (reverse; position 2587-2613), 5'-GTTGACTCAGATTGGTTGCAC3' (forward; position 2519-2539). 5'-GTATGTCATT GACAGTCCAGC-3' (reverse; position 3305-3324).

The nested PCR products were purified by the Concert ${ }^{\mathrm{TM}}$ Rapid PCR purification system (Life Technologies), and quantified by means of $2 \%$ agarose gel running along with low DNA Mass Ladder ${ }^{\mathrm{TM}}$ (Life Technologies). Sequencing of both PR (codon 1 to 99) and RT (codon 1236) genes relative to HXB2 was performed employing fluorescent dideoxynucleotides (ddNTPs) from ABI Prism BigDye $^{\mathrm{TM}}$ Terminator (ABI) with inner primers resolved on a 377 or 3100 automatic sequencer (ABI). HIV clade was accessed at www.ncbi.gov and the mutation profile (D'Aquila et al. 2002) was analyzed using the Stanford HIV Drug Database (www.stanford.edu). The genotype reports were sent to the respective clinicians. Statistical analysis was made by means of the EpInfo6 program (CDC), using Yates corrected test or Fisher's exact test, two tailed.

\section{RESULTS}

Sequences were obtained from blood donated by 306 patients. The median age of patients was 35 years, interquartile range (IQR 25th, 75th ): 27, 42 years, being $68 \%$ male, and $70 \%$ of them were using three or more ARV regimens. TCD $4^{+}$counts were below 200 cells $/ \mu 1$ in $51 \%$ patients. This population had median TCD4 ${ }^{+}$counts of 206 cell/ $/$ l (IQR 97, 338) at sample collection and included 44 children (up to 12 years old), median age $8(5,9)$, median TCD $4^{+} 415$ cells/ $\mu \mathrm{l}(97,793) ; 16$ adolescents (from 13 to 19 years old), with median age of $15(13,17)$, median TCD $4^{+} 127$ cells/ $\mu 1(31,208)$; and 246 adults, (age above 19 years old), median $37(32,43)$ years old, median TCD4 $4^{+} 190$ cells $/ \mu \mathrm{l}(100,323)$. The median plasma HIV RNA $\log _{10}$ was 4.79 copies $/ \mathrm{ml}(4.32,5.21)$, being in children 5.3 copies $/ \mathrm{mL}$ $(4.90,5.52)$, in adolescents 5.20 copies $/ \mathrm{ml}(4.71,5.42)$ and in adults 4.63 copies $/ \mathrm{ml}(4.20,5.07)$.

Most of the patients had a long exposure to ARV therapy, as $28 \%$ of them started therapy before 1997, with a median time of ARV exposure of five years (IQR: 3.3, 6.2 years). Over half of the patients (51\%) had previously been exposed to single drug class of nucleoside reversetranscriptase inhibitors (NRTI), but only $2 \%$ were on dual NRTI therapy at sample collection for genotype. The percentage of cumulative number of treatment regimens to which the patients have been exposed to at sample col- lection was $12 \%$ at first regimens, $28 \%$ at second, $23 \%$ at third, $14 \%$ at fourth, $14 \%$ at fifth and $9 \%$ using their sixth or more therapy regimens. Eighty-one different ARV regimens were observed at the time of sample collection for genotype testing, and the most common regimens were AZT/3TC/nelfinavir, 3TC/D4T/nelfinavir, and AZT/3TC/ efavirenz, which were in use by nearly one third of patients. Fig. 1 shows the ARV class composition of regimens used by patients at time of genotype testing according to the cumulative number of regimens used, as $\mathrm{T} 1$, for exposure to one regimen at collection, up to $\mathrm{T}^{+}$, for six or more ARV regimens.

The mean number of viral mutations at the principal codons of PR and RT genes is presented in Fig. 2, according to number of ARV regimens used by subjects. Fig. 3 depicts the percentage of mutations at codons related to ARV resistance according to the number of regimens used by patients: 9a) for protease inhibitors (PI); (b) for NRTI; and (c) to non-nucleoside NNRTI. At least one thymidine-associated mutation (TAM), that is, ARM at posi-

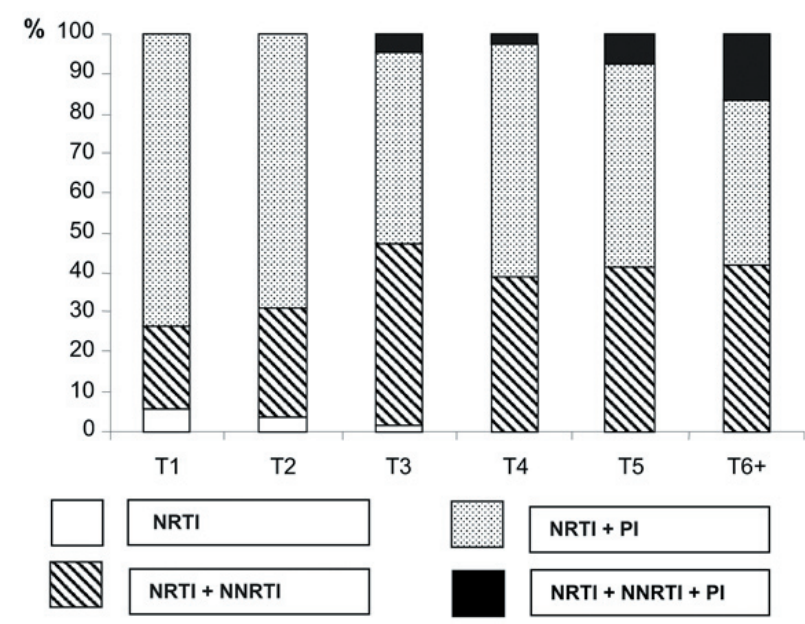

Fig. 1: antiretroviral class composition (\%) of regimens, as single class (one or more) nucleoside reverse-transcriptase inhibitors (NRTI), combination of NRTI plus a non-nucleoside reverse-transcriptase inhibitors (NRTI + NNRTI); of NRTI + protease inhibitor (NRTI + PI) or a three class combinations, NRTI + NNRTI + PI in use at the time of blood collection for genotype by cumulative number of treatment regimens used up to genotyping, from one treatment regimen (T1) up to six or more regimens (T6+).

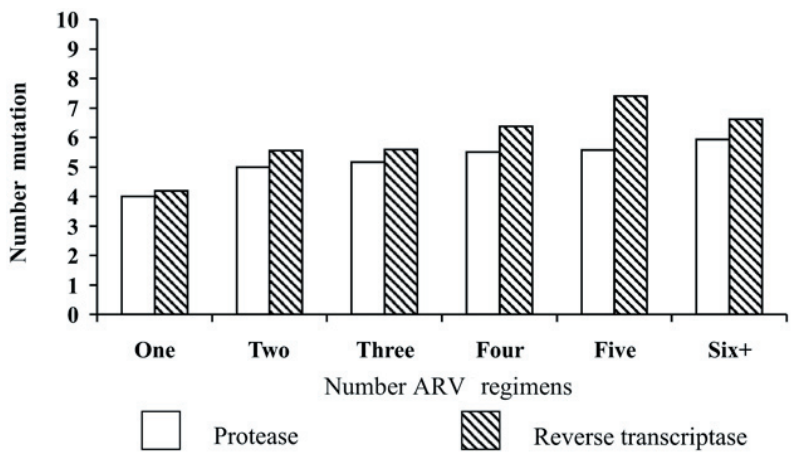

Fig. 2: mean number of antiretroviral resistance mutations (ARM) in patient's HIV-1 isolates by the cumulative number of antiretroviral regimens ( 1 to 6 or more) used during treatment history, at protease and reverse transcriptase genes. 

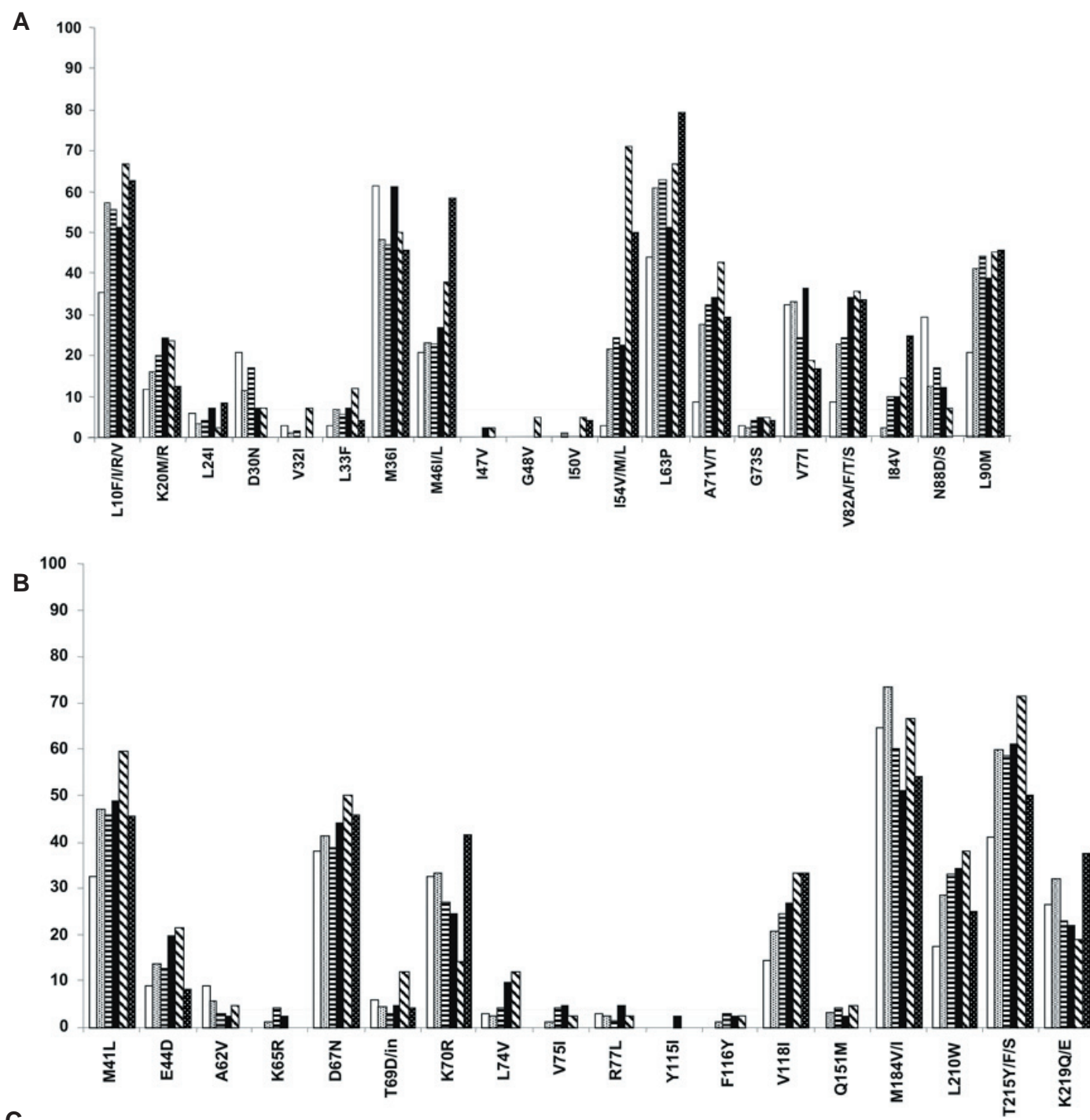

C

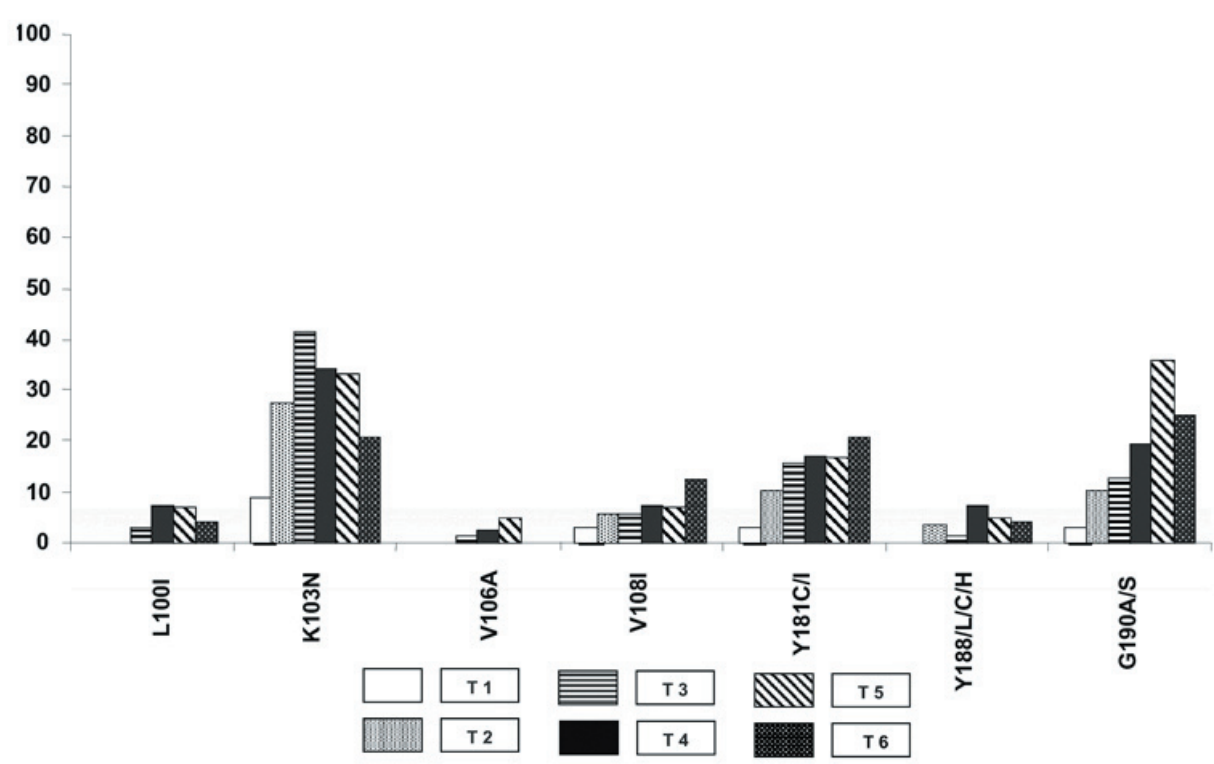

Fig. 3: percentage of antiretroviral resistance mutations (ARM) at selected codons associated to decrease susceptibility (A, to protease inhibitors; $\mathrm{B}$, to nucleoside reverse-transcriptase inhibitors; and $\mathrm{C}$, to non nucleoside reverse-transcriptase inhibitors) antiretroviral class, by number of regimens used, such $\mathrm{T} 1$ to $\mathrm{T} 6+$ for exposure from one to six or more antiretroviral regimens. 
tions $41,67,210,215$ or 219 of RT, was seen in $73 \%$ of isolates, and mutations at residue M184 occurred in 64\% of cases. Multi-nucleoside resistance mutations such Q151M were rare, but significantly associated with mutations at residue V75 ( $\mathrm{p}<0.00003$ ), including not only mutations to isoleucine, but also with the uncommon mutation to threonine. We tried to estimate the pattern of mutation to some of the most used medications in our population. The protease inhibitor (PI) nelfinavir, has reduced the susceptibility associated either to a specific mutation, at residue D30, or to mutations that affect other PI, such as L90M. Among those receiving nelfinavir as the single PI, 68\% had a principal mutation to Nelfinavir. L90 and D30 mutations were observed alone or in combination in the majority of these patients. L90M was more frequent, observed in 33\% of patients, while D30N was noted in $27 \%$, and a combination of both mutations was observed in $6 \%$ of individuals. Mutations at residue V82 were rare in cases exposed only to Nelfinavir as PI, observed in $2 \%$. Taking into account exposure to all PI, the L90M was the foremost mutation, occurring in $41 \%$ of cases, followed by mutations at residue V82 which was detected in $25 \%$ of patients. Other protease inhibitor specific mutations, such as residue I50, were rarely observed.

Agreeing with previous studies (Sabino et al. 1996, Bongertz et al. 2000), the majority of the isolates of our study $(70 \%)$ could be genetically clustered to HIV-1 clade B, with $12 \%$ clustering to HIV-1 clade F. Fig. 4 shows the percentage of HIV-1 clades at PR and RT genes. Evidence of $\mathrm{B} / \mathrm{F}$ recombinant was observed in $18 \%$ of these sequences. Fig. 5 shows the proportion of ARV related mutations at selected codons in protease and reverse transcriptase clustering to references of HIV-1 clade B or HIV1 clade F. No important difference could be observed when major ARV resistance mutations were analyzed, but some polymorphisms were significantly $(\mathrm{p}<0.00002)$ associated to HIV-1 protease clade $\mathrm{B}$ (at residue L63) or clade $\mathrm{F}$ (at residues K20, M36 and L89).
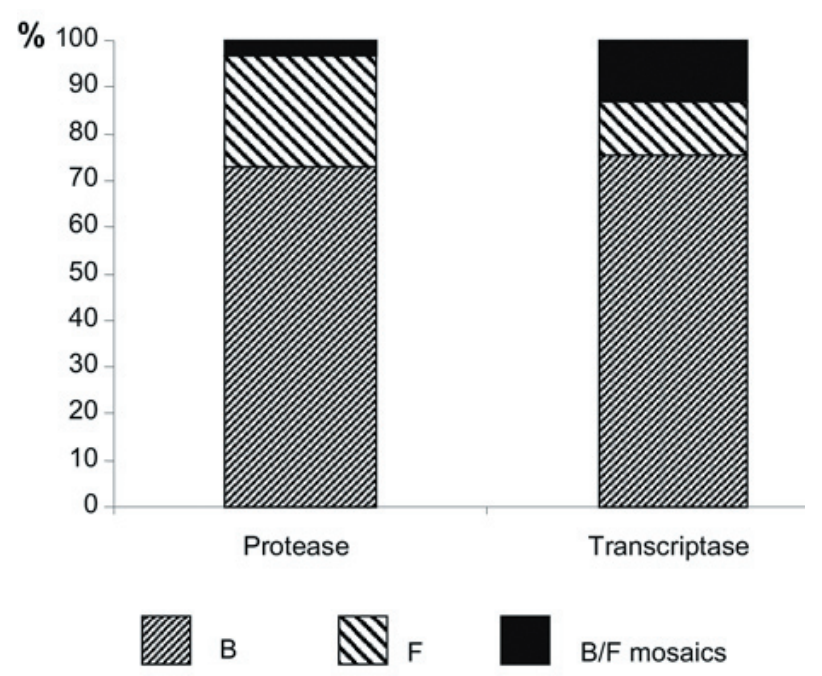

Fig. 4: proportion of HIV-1 protease and reverse transcriptase genomic segments analyzed at the pol region clustering with reference clade sequences. B for HIV-1 clade B, F for HIV-1 clade F1, and $\mathrm{B} / \mathrm{F}$ to HIV-1 mosaics.

\section{DISCUSSION}

The present study describes the ARV mutation profile in clinical samples from patients of São Paulo and nearby cities, and its association to the number of treatment regimens. Some represented municipalities of the state of São Paulo are among the first to introduce highly active antiretroviral therapy (HAART) as part of the public AIDS care service. The exposure to multiple regimens, many of them starting in the pre-HAART era, makes this patients prone to the development of resistance. Patients treated in early 1990s were exposed to one ARV class (NRTI) therapy. Even after 1996, with HAART therapy, some ARV combinations included hard capsule Saquinavir as single PI, situations now known to be associated to sub-optimal regimens. Moreover, adherence to treatment regimen was not a concern incorporated into clinical practice until recently.

The mean number of mutations observed in these patient populations is high and increases with the number of regimens used, and it reflects the high prevalence of ARM at individual codons (Fig. 3). Although an increase is observed with the increase of regimens used, the relationship is not direct and the mean number of ARM is already elevated even in patients failing to first or second regimen for PI, it increases from a mean of four ARM for those failing a first ARV regimen to up to six ARM for those previous exposed to six or more regimens at genotype. At RT we can observe similar trend (Fig. 2). The mean number of total mutations, as well as ARM at key codons (as M184, T215 at RT or L90 at PR) is high even after initial regimens, a fact that makes rescue therapy increasingly difficult. Not only the total number of mutations, but an extensive mutation profile was observed in this population. Moreover, previous ARV exposure itself, even if without detectable resistance mutation, as well as other factors, may play a role in modulating the response to rescue therapy. Historical, clinical and laboratory evidence may not be sufficient to rule out a possible antiviral response to rescue therapy of heavily pretreated patient population (Montaner et al. 2001). In this population, $60 \%$ of the subjects where exposed to three or more antiretroviral regimens. The benefit of resistance genotype test to rescue therapy in this setting is unclear (Loutfy $\&$ Walmsley 2002, Valer et al. 2004) but a benefit cannot be ruled out. The lack of follow-up information does not allow us to address this question in this study and results of other studies (Clevenbergh et al. 2000) may not apply to our population. Studies are needed to access the benefit of the test in these situations and to evaluate the cost-effectiveness of the genotype test as a guide to rescue therapy.

Although no difference in prevalence of major, principal resistance mutations was observed in comparing clade HIV-1 B and non-B (almost exclusively HIV-1 F1 in our population), remarkable differences in the prevalence of some secondary mutations were evident. The impact of polymorphisms of non-B HIV-1 in resistance evolution must be properly evaluated. The emergence of numerous drug class-related ARM, as TAM, detected in $73 \%$ of patients or general PI resistance mutations (mutations at 


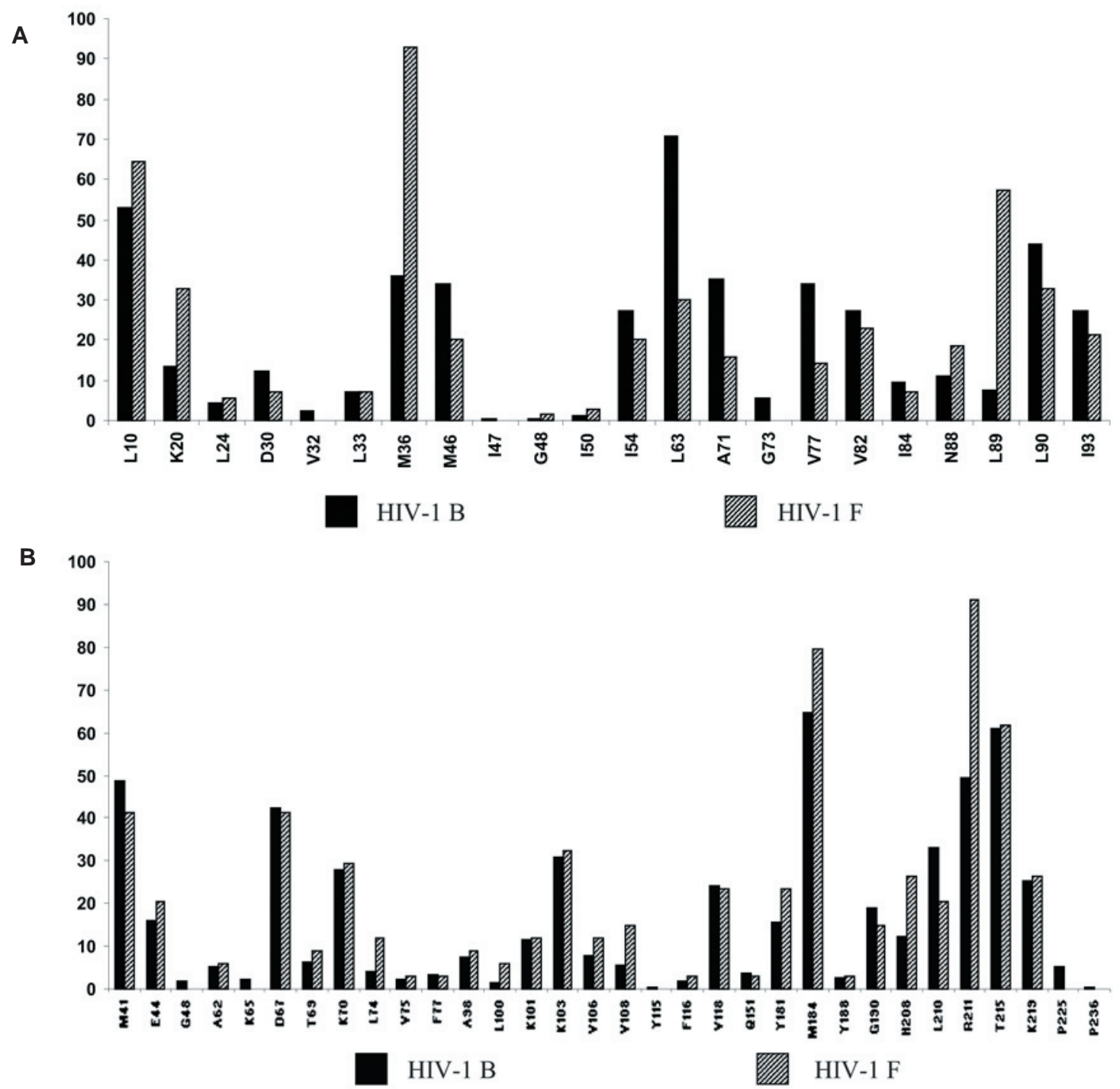

Fig. 5: percentage of antiretroviral related mutations at selected codons. A to protease and B to reverse transcriptase genes clustering to references of HIV-1 clade B or HIV-1 clade F.

V82, I84 or L90 residues that cause high level resistance to two or more protease inhibitors), detected in 54\% of cases, may probably be a major impediment for a successful salvage regimen. The presence of both TAM and principal mutation to PI, documented in $47 \%$ of subjects, limits further the formulation of salvage therapy.

As for individual ARM, L90M mutation was the most frequently observed at the protease $(41 \%)$, and mutations at the M184 of RT in $64 \%$ of subjects. The former decreases susceptibility of most available protease inhibitors, and the latter, albeit associated with increased susceptibility to AZT when ARM to this drug are present, probably have this effect minimized by the large number of TAM in most patients. More importantly, the mutation affected one of the most commonly used NRTI in this population, that is, the 3TC. Some mutations uncommon in vivo, such as V75T, were observed in $2.3 \%$, and the majority of them along with multi-nucleoside mutation Q151M. These resistance profiles are more pronounced in children, but the difference has not been significant. The role of mutation profile in viral fitness (Nijhuis et al. 2001, Antinori et al. 2001) and the difficulty in providing adequate regimens options must be taken into account in the decision to recommend a salvage therapy aiming viral suppression after repeated therapeutic failures (Deeks et al. 2000, Murphy et al. 2001). This is an increasingly public health issue that needs better research evaluation.

No formal comparison of ARV regimen potency or the association of these regimens to therapy failure can be assessed in the present study design. The proper sequencing, drug combinations and strategies to provide an adequate rescue therapy could not also be evaluated. The lack of adequate information on adherence to medication precludes a proper correlation of regimen and the emergence of resistance. It is interesting to note that, many of regimens in use and on virological failure included ARV drugs mostly represent the current international acquisition budget by the Brazilian AIDS Program, being $63 \%$ of 
the cost in 2003 due to three brand drugs. These are popular regimens and we cannot infer any relationship to the virological failure in this uncontrolled study. However, the role of actual biological or pharmacological characteristics in selecting these drugs as part of HAART regimens, as compared to the role played by drug company marketing strategies is an important issue that need to be addressed. Our study cannot address these issues, however the possibility in using an alternative, generic drugs regimens, that may provide similar or even better efficacy, should be evaluated in clinical trials for these may delineate valuable implications for the long-term sustenance of the Brazilian ARV Access Program.

\section{REFERENCES}

Antinori A, Liuzzi G, Cingolani A, Bertoli A, Di Giambenedetto S, Trotta MP, Rizzo MG, Girardi E, De Luca A, Perno CF 2001. Drug resistance mutation of HIV-1 in patients exhibiting increasing CD4 cell counts despite virological failure of HAART. AIDS 15: 2325-2327.

Bongertz V, Bou-Habib DC, Brigido LF, Caseiro M, Chequer PJ, Couto-Fernandez JC, Ferreira PC, Galvao-Castro B, Greco D, Guimaraes ML, Linhares de Carvalho MI, Morgado MG, Oliveira CA, Osmanov S, Ramos CA, Rossini M, Sabino E, Tanuri A, Ueda M 2000. HIV-1 diversity in Brazil: genetic, biologic, and immunologic characterization of HIV-1 strains in three potential HIV vaccine evaluation sites. Brazilian Network for HIV Isolation and Characterization. J Acquir Immune Defic Syndr 23: 184-193.

Casseb J, Pereira Junior LC, Silva GL, Medeiros LA 1999. Decreasing mortality and morbidity in adult AIDS patients from 1995 to 1997 in Sao Paulo, Brazil. AIDS Patient Care STDS 13: 213-214.

Clevenbergh P, Durant J, Halfon P, del Giudice P, Mondain V, Montagne N, Schapiro JM, Boucher CA, Dellamonica P 2000. Persisting long-term benefit of genotype-guided treatment for HIV-infected patients failing HAART. The Viradapt Study: week 48 follow-up. Antivir Ther 5: 65-70.

D’Aquila RT, Schapiro JM, Brun-Vezinet F, Clotet B, Conway B, Demeter LM, Grant RM, Johnson VA, Kuritzkes DR, Loveday C, Shafer RW, Richman DD, International AIDS Society-USA 2002. Drug Resistance Mutation in HIV-1. Topics in HIV Medicine 10: 21-25.

Deeks SG, Barbour J, Matin J, Swanson M, Grant RM 2000. Sustained CD4 $\mathrm{T}$ cell response after virologic failure of protease inhibitor based regimens in HIV infected patients.
J Infect Dis 181: 946-953.

Larder BA, Kemp SD 1989. Multiple mutations in HIV-1 reverse transcriptase confer high-level resistance to zidovudine (AZT). Science 246: 1155-1158.

Loutfy MR, Walmsley SL 2002. Salvage antiretroviral therapy in HIV infection. Expert Opin Pharmacother 3: 81-90.

Marins JR, Jamal LF, Chen SY, Barros MB, Hudes ES, Barbosa AA, Chequer P, Teixeira PR, and Hearst N 2003. Dramatic improvement in survival among adult Brazilian AIDS patients. AIDS 17: 1675-1682.

Ministério da Saúde, CN-DST/Aids, Brazil 2004. Recommendations for Antiretroviral therapy in HIV-infected adults and adolescents - 2002/2003. Available at http://www. aids.gov.br/final/tratamento/consenso_english_2003.htm.

Montaner JSG, Harrigan PR, Jahnke N, Raboud J, Castillo E, Hogg RS, Yip B, Harris M, Montessori V, O'Shaughnessy MV 2001. Multiple drug rescue therapy for HIV-infected individuals with prior virologic failure to multiple regimens. AIDS 15: 61-69.

Murphy EL, Collier AC, Kalish LA, Assmann SF, Para MF, Flanigan TP, Kumar PN, Mintz L, Wallach FR, Nemo GJ; Viral activation transfusion study investigators 2001. Highly active antiretroviral therapy decreases mortality and morbidity in patients with advanced HIV disease. Ann Intern Med 135: 17-26.

Nijhuis M, Deeks S, Boucher C 2001. Implications of antiretroviral resistance on fitness. Curr Opinion in Infec Dis 14: $23-28$.

Sabino EC, Diaz RS, Brigido LF, Learn GH, Mullins JI, Reingold AL, Duarte AJ, Mayer A, Busch MP 1996. Distribution of HIV-1 subtypes seen in an AIDS clinic in Sao Paulo City, Brazil. AIDS 10: 1579-1584.

Shafer RW, Kozal MJ, Winters MA, Iversen AK, Katzenstein DA, Ragni MV, Meyer WA, Gupta P, Rasheed S, Coombs R 1994. Combination therapy with zidovudine and didanosine selects for drug-resistant human immunodeficiency virus type 1 strains with unique patterns of pol gene mutations. J Infect Dis 169: 722-729.

Valer L, Gonzalez de Requena D, de Mendoza C, MartinCarbonero L, Gonzalez-Lahoz J, Soriano V 2004. Impact of drug levels and baseline genotype and phenotype on the virologic response to amprenavir/ritonavir-based salvage regimens. AIDS Patient Care STDs 18: 1-6. 